\title{
Algunas relaciones entre algoritmos y resolución de problemas
}

Recibido:30-06-2010|Aceptado. 20-12-2010

Some relationships between algorithms and problem solving

Jaime Fonseca González*

Brigitte Johana Sánchez Robayo**

Resumen: Como parte del estudio realizado en la tesis de maestría "Uso de algoritmos en la resolución de problemas sobre isometrías del plano. Un estudio de caso", se han identificado diversas formas en que se relacionan los algoritmos con la resolución de problemas, no sólo como procesos mecánicos, sino como fuente de información en el planteamiento de estrategias al resolver un problema, de modo que surgen algunas maneras en que se da esta relación, ejemplificando cada una de ellas con problemas cuyo objeto matemático central son las isometrías del plano.

\begin{abstract}
As part of the study carried out in the Master Program entitled "Use of algorithms in Solving Problems on Plane Isometries: A Case Study" the authors have identified new ways in which algorithms are related to resolution of problems, not only as a mechanical process but as a source of information in the planning of strategies to solve problems, thus raising some ways in which this relationship is given, illustrating each one of them with problems which central mathematical objects are the plane isometries.
\end{abstract}

Palabras clave: algoritmos, resolución de problemas, isometrías del plano.
Keywords: Algorithms, Problem Solving, Plane Isometries.

* jaimejaimef@hotmail.com

**bjsanchezr@udistrital.edu.co 


\section{Introducción}

El papel de los algoritmos en el estudio de las matemáticas se ha venido replanteando como producto de los resultados de múltiples investigaciones, al identificar en ellos concepciones sobre distintos objetos matemáticos y el potencial que tiene la creación de los mismos en la enseñanza de las matemáticas. También se han estudiado maneras en que pueden ser enseñados algunos algoritmos que se presentan al estudiar temáticas de diversas ramas de las matemáticas, como la aritmética y el álgebra.

Como resultado del proyecto anteriormente mencionado, se han determinado diversas condiciones en las que se pueden presentar usos de algoritmos en la resolución de problemas sobre isometrías, tales que proporcionan información para que el resolutor observe y logre determinar nuevas relaciones entre los elementos del problema. A continuación se presentan algunas de las formas identificadas para usarlos, así como la caracterización de tales usos y el ejemplo de algunas evidencias observadas durante el estudio realizado.

\section{Algoritmos y resolución de problemas}

Por medio de la resolución de problemas, los estudiantes pueden realizar las mismas tareas que el matemático cuando resuelve problemas de la disciplina y experimentar el potencial y utilidad de las matemáticas en el mundo que les rodea. Esta es una de las razones por las que la resolución de problemas se ha introducido desde hace varios años en la educación matemática. Uno de los trabajos fundamentales en la enseñanza de la resolución de problemas fue presentado por George Polya en 1945 en el libro How to solve it?, en el cual se ilustra por pri- mera vez un camino hacia la enseñanza de la resolución de problemas y se ha considerado base para muchas investigaciones cuyas conclusiones han permitido madurar las ideas de este autor.

Para el presente caso, esta teoría permitió relacionar de manera directa la utilización de algoritmos en la resolución de problemas de geometría, particularmente de isometrías del plano. Como primera instancia, es necesario especificar algunos aspectos de los algoritmos y de la resolución de problemas, de tal forma que la conjugación de los mismos permita formular detalladamente tal relación.

\section{Algoritmos}

El algoritmo es entendido como una secuencia finita de pasos, exenta de ambigüedades, que lleva a la solución de un problema dado. Los pasos de un algoritmo toman de base un conjunto de datos iniciales, con los cuales se determina un conjunto de datos finales que corresponden a la solución del problema dado.

Los algoritmos deben cumplir las siguientes especificaciones:

- Precisión: en el algoritmo se debe indicar el orden en el que se ejecutan cada uno de los pasos propuestos.

- Bien definido: si se ejecuta el algoritmo varias veces con los mismos datos iniciales, se deben obtener siempre los mismos resultados.

- Finito: la ejecución del algoritmo debe terminar en algún momento, es decir que la cantidad de pasos del algoritmo debe ser finita. 
- Corrección: el algoritmo debe solucionar el problema para el cual fue diseñado, ello implica que debe verificarse.

Aunque la noción de algoritmo es más usada en procedimientos numéricos y algebraicos, siguiendo a Martin (1997), ésta también se encuentra en geometría, específicamente, las construcciones geométricas son un tipo particular de teorema que puede catalogarse como un algoritmo geométrico: "una construcción es el tipo especial de teorema que es también un algoritmo" (p. 2).

En geometría, la palabra construcción tiene dos significados: de algoritmo geométrico o de dibujo que ilustra un teorema. En la investigación se emplearon los dos significados, utilizando el término algoritmo para hacer referencia al primero y reservando la palabra construcción al segundo.

\section{Sobre la resolución de problemas}

Considerando diversas definiciones y la caracterización que realizó Polya, se entiende problema como una situación que tiene unos datos, condiciones y una incógnita.

Según Polya (1965-2002 p. 161), la incógnita"recibe también el nombre de 'quasitum,' lo que se busca, o lo que se pide". En este sentido, la incógnita corresponderá al objetivo, meta o estado final de la situación. La condición es la información que relaciona los datos con la incógnita, y los datos son expresiones generales que describen los objetos que intervienen en el problema. Frecuentemente, la condición se divide en varias partes que a su vez son condiciones del problema, sin embargo, para evitar ambigüedades, cada una de estas partes será conocida como cláusula.

Un problema será insoluble si todos sus elementos son completamente nuevos o no relacionales con un problema ya solucionado, "Es prácticamente imposible imaginar un problema completamente nuevo, que no se parezca en nada a otro o que no tenga ningún punto en común con un problema anteriormente resuelto; por lo demás, si un tal problema existiese sería insoluble" (Polya, 1965-2002. p. 66).

En su propuesta, Polya presenta cuatro tipos de problemas: problemas por resolver, problemas por demostrar, problemas de rutina y problemas prácticos. Aunque esta tipología fue adoptada para la investigación, los problemas prácticos no se consideraron en el diseño de los instrumentos de recolección de información, debido a la falta de claridad que se presenta en sus elementos y a la cantidad de datos y condiciones que deben tenerse en cuenta para su resolución.

Problemas por resolver. Los problemas por resolver tienen mayor importancia en las matemáticas elementales y su objetivo es encontrar la incógnita del problema. Dentro de este tipo de problemas, se pueden encontrar problemas teóricos o prácticos, abstractos o concretos, o simplemente, problemas cuyas incógnitas sean de cualquier tipo.

Una manera de resolver este tipo de problemas es movilizando conocimientos ya adquiridos; se deben buscar problemas que estén ligados al original por medio de la generalización, particularización o analogía ${ }^{1}$. Además, Polya recomienda considerar por separado los datos o las diversas partes en las que se puede dividir una condición.

Problemas por demostrar. Este tipo de

1 Los términos generalización, particularización y analogía serán abordados más adelante. 
problemas tiene como objetivo "mostrar de modo concluyente la exactitud o falsedad de una afirmación claramente enunciada" (Polya, 1965-2002 p. 161). Además, cumplen la definición de problema, ya que la hipótesis contiene los datos y las condiciones, y la demostración corresponde a la incógnita.

Problemas de rutina. Hacen referencia a todos aquellos problemas que pueden resolverse sustituyendo datos en problemas ya resueltos o siguiendo paso a paso la secuencia de algún ejemplo. “Un problema se convierte de rutina, cuando ya se han solucionado varios problemas similares, cambiando únicamente algunos datos."(Polya, 1965-2002 p. 163).

Problemas prácticos. Estos problemas se caracterizan por la gran cantidad de datos y condiciones, la falta de claridad al determinar las incógnitas, los datos y las condiciones, y al identificar los conocimientos y conceptos necesarios para solucionarlos.

\section{Etapas en la resolución}

\section{de problemas}

Aunque es claro que no existe un método que garantice la solución de cualquier problema de matemáticas, Polya sugiere un método basado en cuatro etapas:

- Comprender el problema. Inicialmente, se debe tener claro el objetivo a alcanzar. Siguiendo a Polya, no se pueden realizar acciones y reflexionar sin antes haber comprendido el propósito deseado. Para comprender el problema, debe familiarizarse con él, leyendo el enunciado, visualizándolo como un todo, para luego aislar las principales partes del problema, centrar la atención en cada una de ellas y combinarlas entre sí, para establecer las relaciones que puedan existir.
- Concebir un plan. En esta etapa se considera el problema desde varios puntos de vista y se busca relacionarlo con los conocimientos previos, problemas resueltos y los teoremas demostrados, de manera tal que se obtenga la solución del problema. Para ello, se debe examinar de manera iterada los detalles del problema, siempre desde visiones distintas, en busca de un nuevo significado o de problemas auxiliares que den un lineamiento de la resolución del problema original.

- Se ha concebido un plan cuando se tiene una idea, así sea grosso modo, de los cálculos, razonamientos o construcciones que permitirán determinar la incógnita. Esta etapa puede ser larga, compleja y es lo esencial en la obtención de la solución de un problema (Polya, 1965).

- Ejecutar el plan. Una vez asegurada la comprensión del problema y establecida una línea general proporcionada por el plan, éste se debe ejecutar prestando atención a los detalles para perfeccionarse y lograr determinar la solución. Si el problema es muy complejo, al igual que el plan para resolverlo, es recomendable expresar el problema como composición de problemas más pequeños, cuyas soluciones conformarán la solución general del problema original.

Debido a que el plan da una idea general de los pasos para la resolución, debe examinarse uno a uno, que todos los detalles coincidan con lo esperado en el plan. Adicionalmente, el orden en que se revisen y ejecuten los detalles del plan, es fundamental para lograr una sistematización de la solución, especialmente si el problema es complejo (Polya, 1965-2002). 
- Examinar la solución obtenida. Aún cuando se haya obtenido una solución y se tenga claro el proceso realizado, se debe reexaminar el resultado y el plan ejecutado, de tal manera que se pueda refinar y obtener una mejor comprensión de la solución. Esta fase es fundamental, pues aunque en la fase de la ejecución del plan se clarificó cada uno de los pasos del proceso, no pueden descartarse errores, especialmente si el problema es largo y complejo. En esta etapa se debe examinar con mucho detenimiento el método que ha llevado a la solución, tratando de aplicarlo a otros problemas; realizando esto posiblemente se encontrará una mejor solución y se descubrirán algunos detalles y relaciones importantes entre las principales partes del problema.

\section{Relaciones entre algoritmos y resolución de problemas}

Posterior a comprender el problema, en la segunda fase del proceso de resolución de problemas propuesto por Polya, se concibe un plan, es decir, se plantea una idea, así sea grosso modo, de los cálculos, razonamientos o construcciones que permitirán determinar la incógnita; si durante las fases de ejecución del plan y verificación de la solución obtenida (tercera y cuarta fase), es posible perfeccionar ${ }^{2}$, sistematizar y eximir de ambigüedades un conjunto finito de pasos que den solución al problema, entonces éste tiene, al menos, un algoritmo que permite determinar su solución y que se denomina algoritmo de solución. La precisión del algoritmo se

2 Al perfeccionar los pasos de solución del problema, la persona culmina dicho proceso garantizando que no haya errores y que la forma en que se presenta, es la óptima que ha podido encontrar. garantiza en la sistematización y perfeccionamiento del conjunto finito de pasos y la corrección está inmersa en la solución misma del problema.

Cuando existe un algoritmo de solución para un problema, se da una correspondencia entre los datos, la incógnita y las condiciones del problema, con los datos iniciales, los datos finales y los pasos del algoritmo, respectivamente.

Un ejemplo de esta correspondencia, se evidencia en el problema de trasladar un punto $X$, según la distancia de dos puntos $P$ y $Q$ en la dirección de $P$ a $Q$. Dicha traslación se ha denotado $[\overrightarrow{\mathrm{PQ}}]$, donde la imagen de $X$ por $[\overrightarrow{\mathrm{PQ}}]$ corresponde a un punto $X^{\prime}$ tal que el polígono $Q P X X^{\prime}$ es un paralelogramo.

Ejemplo. Trasladar el punto $X$ a través de $[\overrightarrow{\mathrm{PQ}}]$

(Ver Tabla 1).

En la propuesta realizada por Polya, se sugieren diversas preguntas, procedimientos y medios que permiten resolver un problema mediante la determinación de problemas auxiliares más sencillos o de solución conocida. Específicamente, propone la generalización, particularización, analogía, descomposición y recomposición, análisis y síntesis, e intercambio entre las partes del problema, como procedimientos para variarlo y obtener uno auxiliar, que eventualmente permita encontrar la solución del problema original.

Particularmente, cuando se tiene un problema a resolver $A$ y un problema auxiliar $B$ que tiene un algoritmo que permite solucionarlo, el algoritmo puede ser usado en la resolución de $A$, ya sea encontrando relaciones entre los datos y las incógnitas, copiando total o parcialmente el algoritmo 
$T \in \triangle \Delta$ Tecné, Episteme y Didaxis

No. $28 \cdot$ Segundo semestre de $2010 \cdot$ pp. $73-87$

Tabla 1. Traslación del punto $X$ según la distancia de dos puntos $P$ y $Q$

\begin{tabular}{|c|c|}
\hline Problema & Algoritmo \\
\hline Datos: punto $X,[\overrightarrow{\mathrm{PQ}}]$ & Datos iniciales: punto $X,[\overrightarrow{\mathrm{PQ}}]$ \\
\hline \multirow{5}{*}{$\begin{array}{l}\text { Condición: el punto } \\
\text { resultante, debe ser la } \\
\text { traslación de } X \text { a través } \\
\text { de }[\overline{\mathrm{PQ}}] \text {; es decir, } \\
\text { debe ser el punto } \\
X^{\prime} \text {, tal que existe el } \\
\text { paralelogramo } Q P X X^{\prime} \text {. }\end{array}$} & Pasos de un algoritmo de solución \\
\hline & Trazar una recta $m$ paralela a la recta $P Q$ por $X$. \\
\hline & Trazar una recta / paralela a la recta $P X$ por $Q$. \\
\hline & Construir el punto de intersección entre las rectas $m$ y $l$. \\
\hline & $\begin{array}{l}\text { El punto encontrado será } X^{\prime} \text {, que corresponde al cuarto vértice } \\
\text { del paralelogramo } Q P X X^{\prime} \text {, es decir, es }[\overrightarrow{P Q}](X)=X^{\prime} \text {. }\end{array}$ \\
\hline $\begin{array}{l}\text { Incógnita: } \\
{\left[\mathrm{PQ}^{\prime}(\mathrm{X})=\mathrm{X}^{\prime}\right.}\end{array}$ & Datos finales: $[\overrightarrow{P Q}](X)=X^{\prime}$ \\
\hline
\end{tabular}

o aplicándolo en la detección de otros datos o algoritmos auxiliares que proporcionen más información para la solución de $A$. En este caso, se dirá que se ha usado el algoritmo de solución del problema $B$ para solucionar el problema original $A$.

\section{Algunos usos de algoritmos al resolver problemas sobre isometrias del plano}

El usar el algoritmo de solución del problema auxiliar $B$, sugiere la introducción de elementos auxiliares que permiten resolver el problema original. En este caso, se dirá que se ha usado un algoritmo para determinar elementos auxiliares.

Las preguntas, procedimientos y medios propuestos pueden conllevar a usar los algoritmos para resolver un problema, ya sea para encontrar un algoritmo si éste existe, o en el caso contrario, simplemente para solucionar el problema. Sin embargo, el algoritmo puede usarse de maneras distintas de acuerdo con las estrategias empleadas para resolver el problema.
En la generalización se pasa del examen de un problema $A$, al examen de un conjunto $B$ de problemas, tal que $B$ contiene al problema $A$. Otro caso de generalización es cuando se pasa del examen de un conjunto limitado de problemas $A$, al examen de un conjunto más extenso de problemas $B$ que incluye al conjunto limitado $A$. En cualquiera de los dos casos, el problema que consiste en solucionar todos los problemas del conjunto $B$, recibe el nombre de problema gene$\mathrm{ral}$, mientras que cada uno de los problemas del conjunto $B$, que es más particular, recibe el nombre de problema particular.

Cuando se desea solucionar un problema particular y se emplea la generalización, el algoritmo de solución del problema general puede ser aplicado para determinar la solución del problema particular. Dado que el problema original es un caso particular, requiere darse un algoritmo particular al problema, el cual puede ser directamente mostrado por el algoritmo que soluciona el problema general. En el caso en que existan algoritmos para la solución del problema general, de éste se puede extraer información para la resolución del problema original. Aquí pueden darse dos usos: 
- Solución inmediata del problema por medio de un algoritmo. Se presenta si se conoce un algoritmo para la solución del problema general y se aplica, sin mayores variaciones, a los datos del problema particular dado.

- Uso del algoritmo de solución de un problema general en la solución de un problema particular. Se presenta cuando, en el intento de solucionar un problema particular, se realiza el proceso de generalización, y si existe, se determina un algoritmo de solución del problema general obtenido; luego este algoritmo puede ser aplicado a los datos del problema particular para solucionarlo, o se puede emplear para establecer relaciones entre los elementos del problema original.

En la particularización se pasa del examen de un conjunto dado de problemas (problema general) al examen de uno de sus subconjuntos (problema particular).

Este procedimiento es aplicado a la resolución de problemas generales, cuando la solución de los problemas particulares permite recopilar información útil o hacer conjeturas para facilitar la solución del problema general. La determinación de aspectos comunes en los algoritmos de solución de los casos particulares pueden ser empleados para la determinación de un algoritmo general para la solución del problema general. En el caso en que existan algoritmos para la solución de los problemas particulares, de ellos se extrae información para la resolución del problema original. Aquí pueden darse dos usos:

- Uso del algoritmo de solución de un problema particular en la solución de un problema general. Se presenta cuando se toma por completo el algo- ritmo que da solución a un conjunto de problemas particulares y se aplica, sin mayores variaciones, a los datos del problema general. Otro caso en el cual se puede presentar este uso, es cuando se utilizan los algoritmos que dan solución a cada uno de los problemas particulares para encontrar relaciones entre los datos y plantear la solución o el algoritmo de solución de un problema general.

- Uso del algoritmo de solución de un problema particular para planear una demostración. Si el problema original es un problema por demostrar, el algoritmo de solución de un problema particular orienta el camino para realizar la demostración deseada.

En la descomposición y recomposición, posterior al planteamiento de un plan, se fija una idea directriz para la solución y se divide el problema, de tal manera que se preste principal atención a sus detalles y ellos se conviertan en un problema auxiliar; luego de solucionar cada uno de ellos, la directriz antes fijada permitirá componer todas las soluciones, para recomponer el problema original y encontrar su solución.

Bajo esta manera de solución, los algoritmos se introducen como una idea general para la resolución del problema original, dividido en procedimientos cuya concatenación asegura la solución del problema; también, si se conoce un conjunto muy limitado de algoritmos para la solución de determinados problemas, es natural que se intente reducir el problema a los problemas conocidos, haciendo del algoritmo general una concatenación de algoritmos más locales.

En este caso, si existen algoritmos de solución para algunos de los problemas 
auxiliares en que fue descompuesto el problema original, es posible tomar los pasos de los algoritmos, los algoritmos completos o relaciones entre los datos, para construir la solución del problema original; de manera específica, el uso de los algoritmos en la resolución del problema original puede presentarse de dos maneras:

- Composición iterada de un mismo algoritmo. Corresponde a la utilización repetida de un mismo algoritmo y se presenta cuando la descomposición del problema conlleva a un conjunto de problemas auxiliares cuyos algoritmos de solución comparten la secuencia de pasos, o que son problemas particulares de un problema general cuya solución se puede obtener mediante la aplicación de un algoritmo.

- Composición de dos o más algoritmos diferentes. Hace referencia a la utilización de dos o más algoritmos que dan solución a los problemas auxiliares en los que fue descompuesto el problema original, donde cada uno de los algoritmos difieren en al menos uno de sus pasos (algoritmos diferentes). Es importante aclarar que si en la solución de un problema se utilizan $n$ algoritmos, donde al menos uno de ellos es diferente de los demás, entonces el uso de los algoritmos corresponderá a la composición de algoritmos diferentes.

En la analogía se utiliza la información que pueda brindar la solución de problemas análogos más sencillos para resolver el problema original. Aunque el uso de la analogía puede generar complicaciones, en múltiples ocasiones la incógnita de un problema análogo más sencillo o el algoritmo mediante el cual se solucionó el problema, pueden aplicarse en el problema original.
"Para resolver un problema que se nos plantea, podemos con frecuencia utilizar la solución de un problema análogo más sencillo, ya sea utilizando su método o su resultado" (Polya, 1945-2002 p. 61); especialmente el algoritmo para determinar la solución puede transferirse para vislumbrar una relación entre los elementos del problema original $y$, con ello, una manera de transferir el algoritmo para la resolución del problema análogo al original.

Si se desea resolver un problema original haciendo uso de la analogía, y el problema o problemas análogos tienen un algoritmo de solución, se pueden encontrar dos maneras diferentes de usar los algoritmos para solucionar el problema original:

- Uso de un algoritmo análogo ${ }^{3}$. Se utiliza el algoritmo de solución del problema análogo más sencillo, ya sea para repetir algunos pasos variando únicamente los datos iniciales, para tomar los datos finales como nuevos datos del problema original o para encontrar relaciones entre los elementos del problema original.

Según Polya, se puede emplear "el método de un problema análogo más sencillo, del cual hemos copiado la solución punto por punto" (1965-2002 p. 61), o emplear el resultado del problema análogo más sencillo, sin preocuparse del modo como se había obtenido dicho resultado o, inclusive, "utilizar a la vez el método y el resultado del problema análogo más sencillo". Con lo cual se puede decir que la persona puede tomar algunos pasos del algoritmo análogo, o todo

3 Un algoritmo análogo es un algoritmo que da solución a un problema análogo al problema original. 
el algoritmo, o simplemente los datos finales. También se considerará que se usa un algoritmo análogo cuando éste no siempre pueda emplearse de inmediato y sea necesario transformar o modificar alguno de sus pasos, para hacerlo extensivo a la resolución del problema original.

En el caso en que se utilice un algoritmo análogo para encontrar elementos auxiliares, este uso no corresponderá al uso de un algoritmo para determinar elementos auxiliares, sino al uso de algoritmo análogo.

Si se busca la solución de un problema $A$, para el cual no se conoce un problema análogo con la misma incógnita, es natural que se intente obtener resultados de problemas análogos, cuya solución es conocida; en este caso, se utilizan los algoritmos análogos que den solución a cada uno de estos problemas, de tal manera, que la información que ellos brinden permitan construir la solución del problema A. Esta manera de usar los algoritmos también hace referencia a la composición de algoritmos. Si los problemas análogos se solucionan usando el mismo algoritmo, entonces será composición iterada de un mismo algoritmo; en caso contrario, corresponderá a composición de dos o más algoritmos diferentes.

Análisis y síntesis. Es claro que si se usan estos dos procesos, el análisis precederá a la síntesis, que es donde se ejecutan los pasos propuestos en el análisis. En esta forma de solucionar problemas, el análisis forma parte de la segunda etapa propuesta por Polya y la síntesis forma parte de la tercera; sin embargo, aunque en la concepción del plan se sugiere cierto orden de pasos, el algoritmo que se propone consiste en realizar los pasos en el orden inverso a como se propuso en el análisis; es aquí cuando se dirá que se ha realizado un algoritmo inverso. Es de aclarar, que el término también se aplica cuando es necesario hacer algunas variaciones a la secuencia de pasos inversa, de tal manera que se logre solucionar el problema original. Si este algoritmo es usado para solucionar un problema, se dirá que se usa un algoritmo inverso.

En el intercambio entre las partes del problema se soluciona un problema a partir de uno auxiliar ya resuelto, donde algunos de los datos y la incógnita del primero, corresponden a la incógnita y algunos de los datos del segundo, respectivamente. Dado que la relación entre la incógnita y los datos se mantiene, si existe un algoritmo que de solución al problema auxiliar, invirtiendo sus pasos es posible solucionar el problema original. En este caso, también se usa un algoritmo inverso.

Cuando se da el uso de algoritmos en la solución de problemas, la utilización de los mismos tiene dos fines esenciales: determinar relaciones entre los datos del problema que vislumbren la solución del problema mismo, o encontrar la incógnita o datos auxiliares que permitan obtener el mismo fin. Por tanto, los usos planteados anteriormente, se han categorizado en:

- Uso de algoritmos para hallar datos. Este uso se presenta cuando se emplean los algoritmos para encontrar la incógnita o datos auxiliares.

- Uso de algoritmos para extraer relaciones entre los datos. Este uso se da cuando los algoritmos permiten extraer relaciones entre los elementos del problema original. 
$T \in \Delta$ Tecné, Episteme y Didaxis

No. 28 • Segundo semestre de $2010 \cdot$ pp. 73-87

Tabla 2. Categorización de los usos de algoritmos

\begin{tabular}{|l|l|}
\hline \multicolumn{1}{|c|}{ Categorías } & \multicolumn{1}{c|}{ Usos de algoritmos } \\
\hline \multirow{4}{*}{$\begin{array}{l}\text { Solución del problema } \\
\text { usando algoritmos para } \\
\text { hallar datos. }\end{array}$} & Solución inmediata del problema por medio de un algoritmo. \\
\cline { 2 - 2 } & Composición iterada de un mismo algoritmo. \\
\cline { 2 - 2 } & Composición de dos o más algoritmos diferentes. \\
\cline { 2 - 2 } & Uso de un algoritmo análogo. \\
\cline { 2 - 2 } & Uso de un algoritmo para determinar elementos auxiliares. \\
\hline \multirow{4}{*}{$\begin{array}{l}\text { Solución del problema } \\
\text { usando algoritmos para } \\
\text { determinar relaciones } \\
\text { entre los elementos del } \\
\text { problema. }\end{array}$} & Paso a un algoritmo general de uno particular. \\
\cline { 2 - 2 } & Paso a un algoritmo particular de uno general. \\
\cline { 2 - 2 } & Uso de un algoritmo análogo. \\
\cline { 2 - 2 } & $\begin{array}{l}\text { Uso del algoritmo de solución de un problema particular } \\
\text { para planear una demostración. }\end{array}$ \\
\hline
\end{tabular}

\section{Evidencias sobre algunos usos de algoritmos}

Algunas evidencias de los usos mencionados se obtuvieron al solicitarles a una profesora de matemáticas de Educación Básica y Media, a un estudiante para profesor de matemáticas y a una estudiante de grado undécimo $\left(11^{\circ}\right)^{4}$ de Educación Media que resolvieran el siguiente problema:

4 Se presentarán algunos ejemplos observados en la profesora de matemáticas y en el estudiante para profesor de matemáticas.
Dado el polígono $A B C D E$ y su imagen $A^{\prime} B^{\prime} C^{\prime} D^{\prime} E$ ' por la translación $P Q$, encuentre el polígono $A^{\prime} B^{\prime} C^{\prime} D^{\prime} E^{\prime}$ a partir de $A B C D E$ aplicando isometrías distintas a la translación $\mathrm{PQ}$.

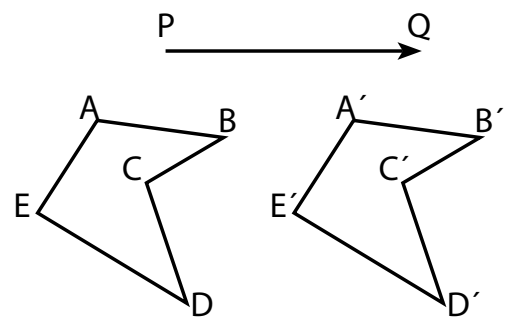

Figura 1. Problema de ejemplo para obtención de evidencias 
La profesora de matemáticas, plantea varias soluciones. La primera, conlleva al siguiente algoritmo de solución:

- Traza la recta $C C^{\prime}$ y construye la recta perpendicular a ella por $C$.

- Refleja el triángulo $A B C$ por la recta construida en el paso anterior.

- Construye la mediatriz de $\overline{C^{\prime}}$ siendo ésta el segundo eje de simetría.
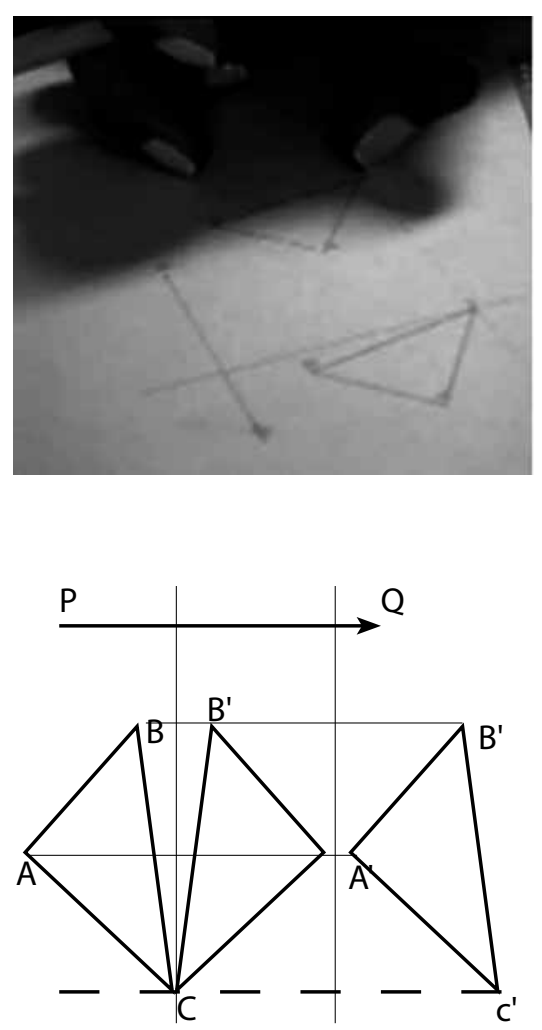

Buscando una segunda solución, la profesora considera la primera y expresa que puede construir una solución distinta, eligiendo el triángulo $A^{\prime} B^{\prime} C^{\prime}$ como inicial (dato) para llegar al triángulo $A B C$ (incógnita).
Bueno, la otra posibilidad fuera que, el eje si estuviera más corrido hacia acá. Lo que pasa es que como voy a obtener esta figura $(\triangle A B C)$, idigo! perdón, obtengo ésta $\left(\triangle A^{\prime} B^{\prime} C^{\prime}\right)$ a partir de ésta $(\triangle A B C)$, ¿cierto? Entonces, yo creo que también se podría hacer. Lo que pasa es que me tendría que devolver y creo que no sería válido. Podría trazar primero un eje acá (recta perpendicular a $\overrightarrow{\mathrm{CC}}^{\prime}$ por $A^{\prime}$ ), ¿cierto? Obtendría otra figura y este eje (recta perpendicular a $\overrightarrow{\mathrm{CC}^{\prime}}$ por (C) quedaría corrido hacia acá. Pero entonces... no sería a partir de ésta $(\triangle A B C)$ obtener esta $\left(\triangle A^{\prime} B^{\prime} C^{\prime}\right)$, sino a partir de éste $\left(\triangle A^{\prime} B^{\prime} C^{\prime}\right)$ obtener éste $(\triangle A B C)$ (Transcripción de lo expresado por la profesora al resolver el problema).

Teniendo en cuenta estas consideraciones, obtiene una segunda solución por medio del siguiente algoritmo:

1. Traza $\overrightarrow{\mathrm{CC}}^{\prime}, \overleftrightarrow{\mathrm{AA}^{\prime}}$ y $\overleftrightarrow{\mathrm{BB}^{\prime}}$

2. Construye la recta perpendicular a $\overrightarrow{\mathrm{CC}^{\prime}}$ por $A$.

3. Refleja $\triangle A^{\prime} B^{\prime} C^{\prime}$ por la recta construida en el paso anterior y nombra $A^{\prime} B^{\prime} C^{\prime}$ a la imagen (aunque la notación es la misma, sólo coincide $A$ ).

4. Construye la mediatriz de $\overrightarrow{\mathrm{BB}^{\prime}}$ ( $B^{\prime}$ es el punto construido en el paso anterior).

5. Las rectas construidas en los pasos 2 y 4 corresponden a los ejes de las reflexiones buscadas.

En las soluciones anteriores, se considera únicamente la aplicación de reflexiones axiales. Esto también se puede evidenciar porque al iniciar la resolución del problema, la profesora expresa "tendría que ser axial, reflexión axial", y al finalizar la segunda solución, dice: "y otra forma de resolverlo aparte de la axial". 
Estas expresiones y las soluciones mismas, muestran que la estrategia planteada por la profesora no se encuentra influenciada por el problema mismo: la iteración de las reflexiones axiales constituye la estrategia considerada por ella, desde que inició la resolución del problema; por esta razón, la intención de resolverlo utilizando únicamente la aplicación de reflexiones axiales, y por ende, el uso repetido del algoritmo asociado, indican que la profesora usó la composición iterada de un mismo algoritmo.

Durante la búsqueda de una tercera solución, la profesora recurre a la composición de dos o más algoritmos diferentes, al expresar su interés en usar composición de diversas isometrías.

Pensaba de pronto en otro, en otra, [] en otro movimiento que me permitiera obtener esa figura, pero tendría que hacer una composición de movimientos para obtener [ ] (quiere obtener $\Delta A^{\prime} B^{\prime} C^{\prime}$ ).

Estoy pensando en si hago una reflexión central aquí (indica punto C) y podría obtener una, hacer una después, o sea, primero hacer una reflexión central teniendo como centro $C$ ¿si? obtenemos una figura y si me daría podría obtener esta figura (posible imagen de $\triangle \mathrm{ABC}$ por [C]), también a partir de ahí o sea, después hacer una reflexión axial" (Transcripción de lo expresado por la profesora al resolver el problema).

Buscando otra forma de solucionar el problema, la profesora centra su atención en la imagen de $\triangle A B C$ por [ $C$, y observa que la segunda isometría que debía usar no era una reflexión axial, sino una reflexión central; también, usó esta imagen y el triángulo $A^{\prime} B^{\prime} C^{\prime}$ para obtener el centro de la reflexión. A partir de allí, obtuvo el siguiente algoritmo de solución:
1. Refleja $\triangle A B C$ por [C] y nombra $A^{\prime} B^{\prime} C$ a su imagen (la notación de los puntos $A^{\prime}$ y $B^{\prime}$ coincide con los puntos $A^{\prime}$ y $B^{\prime}$ del triángulo dado, pero son puntos distintos).

2. Encuentra el centro de la segunda reflexión central, trazando los segmentos $\overrightarrow{C C}^{\prime}, \overrightarrow{\mathrm{AA}^{\prime}}$ y $\overrightarrow{\mathrm{BB}}^{\prime}$ (en los dos últimos segmentos, los extremos son puntos diferentes, según la aclaración de la notación mencionada anteriormente).

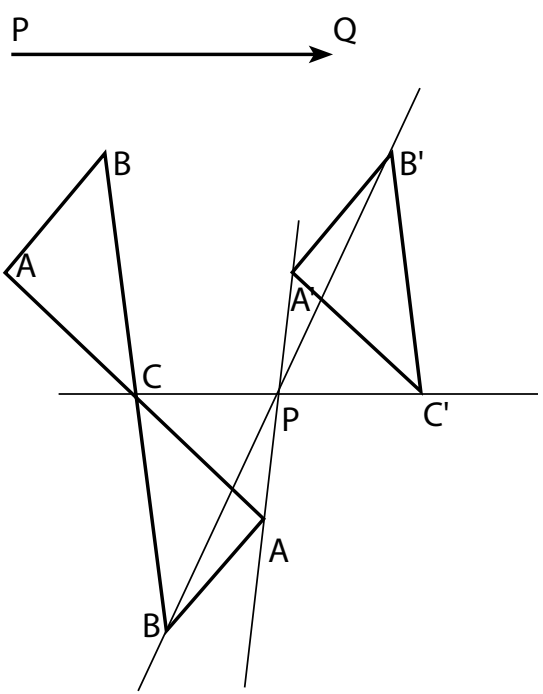

Figura 3. Ejemplo de solución inmediata del problema por medio de un algoritmo

En esta manera de resolver el problema, se puede observar que la profesora lo dividió en dos problemas auxiliares. El primero, encontrar la reflexión del triángulo $A B C$ por $[C]$ y el segundo, determinar la isometría que permite obtener el triángulo $A^{\prime} B^{\prime} C^{\prime}$ de $[C](\triangle A B C)$. Este último tuvo una solución inmediata al encontrar el centro de la reflexión: 
¿Cómo hago para saber que acá? (se refiere a cómo encontrar la isometría que le permite obtener $A^{\prime} B^{\prime} C^{\prime}$ de $\left.[C]\{\triangle A B C\}\right)$, pues tengo que trazar la recta que pasa por A y por A", tengo este acá (indica $A^{\prime \prime}$ ) y el punto de encuentro tendría que ser el centro de la reflexión" (Transcripción de lo expresado por la profesora al resolver el problema).

Por tanto, este problema auxiliar fue solucionado de manera inmediata por medio de un algoritmo (encontrar la isometría dadas la imagen y preimagen).

En la tercera solución del mismo problema (ver figura 4), propuesta por el estudiante para profesor fue:

- Refleja $\triangle A B C$ por [C].

- Identifica el centro de la segunda reflexión central con la intersección de $\overline{\mathrm{CC}^{\prime}}, \overline{[\mathrm{C}](\mathrm{A}) \mathrm{A}}$ y $\overline{[\mathrm{C}](\mathrm{B}) \mathrm{B}}$.
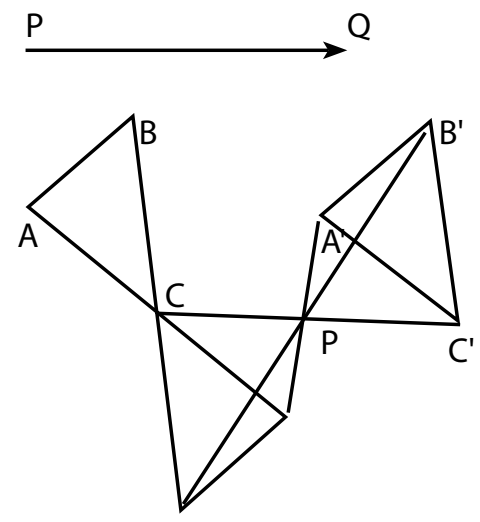

$[P]([c(\pi)])$

$[P(\pi)]([c(\pi)])$

Figura 4. Ejemplo de paso a un algoritmo general de uno particular.
Cuando se le cuestiona por esta solución, afirma:

Estaba pensando en ésta (indica la hoja en donde se encuentra la primera solución). Pues veo también. Estaba viendo esto como una reflexión, como una rotación respecto a algo. Pero no me dio y menos mirándola acá (señala la tercera solución). Entonces empecé a pensar en una rotación, en una rotación acá (señala el punto C), y pues lo que hice fue hacerlo desde el mismo punto del triángulo que había tomado antes (refiriéndose a la primera solución y al punto $C$ ). Hice eso y traté de encontrar un punto y de un momento a otro me fijé fue entre la correspondencia entre los puntos correspondientes $y$, vi que más o menos estaban sobre el mismo lado; tracé y pues, aunque no me dio exactamente, sí supongo que con una construcción un poco más rigurosa y más exacta, sí habría quedado en el mismo punto. $Y$ ya. Fue reflexión central o rotación de 180 (Transcripción de lo expresado por el estudiante para profesor al resolver el problema).

Basándose en la solución anterior, considera otra similar (usando únicamente dos reflexiones centrales) variando el centro de la primera reflexión, y afirma que si en esa solución el procedimiento funciona igual, entonces puede considerar un punto cualquiera sobre el plano y realiza el mismo procedimiento (ver figura 5). En esta solución se puede evidenciar que el estudiante para profesor usa el algoritmo anterior y lo generaliza a cualquier punto del plano, es decir, pasó a un algoritmo general de uno particular.

Pero sí me da (se refiere al intento de hacer dos reflexiones centrales con centros externos a $\triangle A B C$ ), diría que desde cualquier otro punto exterior." 
No sería algo riguroso, pero si sale esto, que ya me salió, me queda como confirmado lo que estoy pensando. Entonces podría haber pensado en una doble reflexión desde cualquier lado, desde cualquier punto, y además como, vuelvo a intentar ahora uno que esté más atrás"(Transcripción).

El algoritmo general obtenido es:

- Considera un punto $R$ del plano.

- Refleja $\triangle A B C$ por R y nombra $\triangle A_{1} B_{1} C_{1}$ a su imagen (el estudiante para profesor sólo nombró $A_{1}$ ).

- Traza ${\overline{A^{\prime}}}_{1}, \bar{B}^{\prime} B_{1}$ y $\overline{C^{\prime} C_{1}}$.

- El punto de intersección entre ${\overline{A^{\prime}}}_{1^{\prime}}$ $\bar{B}^{\prime} B_{1}$ y $\bar{C}^{\prime} C_{1}$, es el centro de la segunda reflexión.

- La imagen de $\mathrm{DABC}$ por la composición de las dos reflexiones obtenidas, corresponde a $D A^{\prime} B^{\prime} C^{\prime}$.

Al confirmar la existencia de algoritmos en geometría, queda como interrogante el establecer otras formas en que se puedan dar los algoritmos en geometría. Adicionalmente, es posible que los algoritmos puedan tener otras aplicaciones al resolver problemas de otras áreas de las matemáticas donde son más usuales, por ejemplo en la aritmética, estadística y álgebra.

\section{Conclusiones}

Algunas maneras en que se usan algoritmos para resolver problemas sobre isometrías son: solución inmediata de un problema por medio de un algoritmo, composición iterada de un mismo algoritmo, composición de dos o más algoritmos diferentes y paso a un algoritmo general de uno particular.
Cuando se comprende un problema y plantea un algoritmo para su solución durante la ejecución del plan, una manera para encontrar otras soluciones, consiste en modificar el primer algoritmo de solución encontrado.

El encontrar diversas maneras en que las personas usan algoritmos en la resolución de problemas sobre isometrías muestra que cuando se explore el campo de la resolución de problemas, un factor a considerar son los algoritmos involucrados.

La resolución de problemas, es hoy uno de los principales enfoques considerados en la enseñanza de las matemáticas, por lo que el objetivo de muchas investigaciones, ha sido la identificación de estrategias de resolución de problemas tanto generales como particulares para diversos tipos de problemas relacionados con diferentes objetos matemáticos, que puedan ser incorporados en la enseñanza de las matemáticas. En este sentido, la identificación de estrategias en las que se incorpora el uso de algoritmos puede formar parte de las propuestas de enseñanza de las isometrías del plano o utilizarse en el análisis de algunos fenómenos en el aula de matemáticas cuando se estudia este objeto matemático.

\section{Bibliografía}

Fonseca, J. y Sánchez, B. (2004) Tutorial de presentación acerca de algunas aplicaciones de los grupos cociente. Trabajo de grado. Universidad Pedagógica Nacional.

Fonseca, J. y Sánchez, B. (2007) Usos de algoritmos en la resolución de problemas sobre isometrías del plano. Un estudio de caso. Tesis de Maestría. Universidad Pedagógica Nacional. 
Jaime, A. y Gutiérrez, A. (1993). Aportaciones a la interpretación y aplicación del Modelo de Van Hiele: la enseñanza de las isometrías del plano. La evaluación del nivel de razonamiento. Universidad de Valencia.

Martin, G. (1998). Geometric Constructions. Springer. New York.

Polya, G. (2002). Cómo plantear y resolver problemas. Trillas.
Puig, L. (1996). Elementos de resolución de problemas. Editorial Comares. Granada, España.

Santos, L. (1997). Resolución de problemas; el trabajo de Alan Shoenfeld: una propuesta a considerar en el aprendizaje de las matemáticas. Revista Educación Matemática. Vol 4 No. 2. Agosto. 